\title{
Pilot study of the antifibrotic effects of the multikinase inhibitor pacritinib in a mouse model of liver fibrosis
}

This article was published in the following Dove Press journal: Journal of Experimental Pharmacology

\author{
Suliman Al-Fayoumi' \\ Taishi Hashiguchi ${ }^{2}$ \\ Yuka Shirakata ${ }^{2}$ \\ John Mascarenhas ${ }^{3}$ \\ Jack W Singer' \\ 'CTI BioPharma, Seattle, WA, USA; \\ ${ }^{2} \mathrm{SMC}$ Laboratories, Tokyo, Japan; ${ }^{3} \mathrm{~T}$ ish \\ Cancer Institute, Icahn School of \\ Medicine at Mount Sinai, New York, \\ NY, USA
}

Background: Fibrotic diseases result from an exuberant response to chronic inflammation. Myelofibrosis is the end result of inflammation in bone, caused by an inflammatory process triggered by production of abnormal myeloid cells driven by mutations affecting the JAK-STAT pathway. Inflammatory cytokine overproduction leads to increased mesenchymal cell proliferation, culminating in fibrosis. Although JAK2 inhibitors, such as the JAK1/2 inhibitor ruxolitinib and the JAK2/FLT3/CSF1R/IRAK1 inhibitor pacritinib suppress abnormal clone expansion in myelofibrosis, ruxolitinib does not appear to prevent or reverse bone-marrow fibrosis in most patients. In two Phase III clinical trials, pacritinib, however, demonstrated improvements in platelet counts and hemoglobin and reductions in transfusion burden in some patients with baseline cytopenias, suggesting it may improve bone-marrow function. Unlike ruxolitinib, pacritinib suppresses signaling through IRAK1, a key control point for inflammatory and fibrotic signaling. Purpose: To investigate potential antifibrotic effects of pacritinib in an animal model of liver fibrosis relevant to the observed course of human disease.

Methods: Pacritinib, negative control (vehicle), and positive control (the angiotensin 2-receptor antagonist and PPAR $\gamma$ partial agonist telmisartan) were assessed in the murine Stelic animal model, which mimics the clinically observed progression from hepatic steatosis to nonalcoholic steatohepatitis, liver fibrosis, and hepatocellular carcinoma. Histopathological analysis used hematoxylin and eosin staining. Body and liver weight changes, nonalcoholic fatty-liver disease activity scores, and plasma cytokeratin 18 fragment levels (a biomarker of hepatic necrosis) were measured.

Results: Pacritinib-treated mice had significantly $(P<0.01)$ reduced fibrotic areas in liver compared to vehicle control and significantly $(P<0.05)$ lower levels of CK18. The antifibrotic effect of pacritinib was comparable to that of telmisartan, but without significant effects on fat accumulation.

Conclusion: These results, the first to demonstrate hepatic antifibrotic effects for pacritinib in an animal model of liver disease, provide preliminary support for potential clinical applications of pacritinib in fibrotic diseases other than myelofibrosis.

Keywords: Janus kinase 2, interleukin 1 receptor-associated kinase 1, colony-stimulating factor 1-receptor kinase, steatosis, myelofibrosis, liver fibrosis

\section{Introduction}

Fibrosis is the end product of chronic inflammation, and has aptly been called "a wound-healing response that has gone out of control."' Common to all fibrotic diseases are activation and proliferation of endothelial cells and fibroblasts in response to inflammation induced by Toll-like receptors that activate IRAK1 causing downstream inflammatory and profibrotic cytokines, including TGF $\beta$. Fibroblasts subsequently
Correspondence: Suliman Al-Fayoum CTI BioPharma, 3101 Western Avenue 600 , Seattle, WA 98I2I, USA

Tel +l 2062724299

Email sal-fayoumi@ctibiopharma.com 
differentiate into myofibroblasts and secrete increasing levels of extracellular matrix proteins including collagens. ${ }^{2}$ This ultimately results in replacement of normal tissues with fibrotic tissue, along with attendant disruption in function that may be limited to single organs (eg, liver fibrosis, pulmonary fibrosis) or involve a systemic process, such as scleroderma. ${ }^{3}$

Myelofibrosis (MF) is a clonal hematopoietic neoplasm originating at the level of multipotential hematopoietic stem cells characterized by an inflammatory response in the bone marrow, leading to progressive fibrosis that impairs normal marrow function, resulting in cytopenias and extramedullary hematopoiesis. ${ }^{4} \mathrm{~A}$ hallmark of bone-marrow response to $\mathrm{MF}$ cells is vascular proliferation ${ }^{5}$ and increasing reticulin and collagen fibrosis in the bone marrow associated, with marked elevation in inflammatory cytokines, including TGF $\beta .^{6} \mathrm{~A}$ central role for JAK-STAT signaling in the pathogenesis of myeloproliferative neoplasms, such as MF, was suggested by the discovery that a gain-of-function mutation in the $J A K 2$ gene $\left(J A K 2^{\mathrm{V} 617 \mathrm{~F}}\right)$ was found in $50 \%-60 \%$ of patients with MF. ${ }^{7-9}$ In light of the unmet need for the treatment of patients with MF, inhibitors of JAK2 have been extensively studied in the clinic.

Ruxolitinib is an oral JAK1/2-kinase inhibitor that has been approved by the US Food and Drug Administration for patients with intermediate- or high-risk MF and those with polycythemia vera who have had inadequate response to or are intolerant of hydroxyurea. ${ }^{10}$ Ruxolitinib has been shown to result in potent reductions in inflammatory cytokineexpression levels in patients with $\mathrm{MF},{ }^{11}$ and its approval in MF was based on data from studies that demonstrated only modest reductions in bone-marrow fibrosis in a fraction of treated patients $(16 \%)$, with fibrosis unchanged $(32 \%)$ or progressing $(19 \%)$ in a higher percentage of patients. ${ }^{12-15}$ Therefore, effective antifibrotic strategies in MF remain an unmet need upon which translational research continues to focus.

Pacritinib is an oral inhibitor of JAK2, FLT3, CSF1R, and IRAK $1^{16-18}$ that has demonstrated activity in clinical studies of patients with MF and other myeloproliferative neoplasms. ${ }^{19-22}$ In Phase II studies, pacritinib was associated with reduction in spleen volume and improvement in symptoms in patients with $\mathrm{MF}^{20}$ and myeloid malignancies ${ }^{22}$ without substantial limiting myelosuppression. In the randomized Phase III PERSIST- ${ }^{23}$ and PERSIST- ${ }^{20}$ studies of pacritinib versus best available therapy in patients with $\mathrm{MF}$, improved hematopoietic function (eg, increased platelet count, increased hemoglobin, reduced transfusion burden) was noted in patients with baseline cytopenias, suggesting restoration in marrow function possibly due to an effect in part on bone-marrow fibrosis.

The kinase profile of pacritinib was evaluated in a kinomewide screening study and demonstrated - in addition to its effect on JAK2 - that it potently inhibits phosphorylation of IRAK1 at an $\mathrm{IC}_{50}<20 \mathrm{nM} \cdot{ }^{18}$ IRAK1 is a kinase situated at a critical juncture of inflammatory signaling from Toll-like receptors and cellular signaling by IL1 leading to downstream activation of both p38 MAPK and p-ERK. The biomarker profile of pacritinib was determined in a panel of human primary cell-based systems designed to model various disease states, including inflammation and fibrosis. ${ }^{24}$ At physiologically relevant levels in a system modeling T-cell-dependent B-cell activation, pacritinib robustly reduced levels of the proinflammatory cytokines soluble IL6 (sIL6), TNF $\alpha$, sIL17A, and sIL17F. Along with TGF $\beta$, IL6 is crucial to promoting $T_{H} 17-$ cell differentiation. ${ }^{25}$ These cells in turn secrete IL17A and IL17F, which promote neutrophil recruitment and infiltration, leading to inflammation and fibrosis. ${ }^{26,27}$ IL17 also stimulates macrophages to produce proinflammatory cytokines. ${ }^{28}$ In addition to its effects on biomarkers, pacritinib has been found to have an antiproliferative effect on endothelial cells and fibroblasts. ${ }^{24}$ Inhibition of several key kinases involved in the elaboration of proinflammatory cytokines, particularly IL17A, and the noted antiproliferative effects provide a strong rationale for examining whether pacritinib is able to modify the fibrotic process in MF. Since clinical assessment of this hypothesis requires invasive procedures in clinical trials of extended duration, this was tested in a standard, well-characterized preclinical model of a fibrotic disease.

Liver fibrosis is a response to chronic hepatocyte injury ${ }^{29}$ that may progress to end-stage cirrhosis. ${ }^{30,31}$ Although it is potentially reversible, ${ }^{32}$ there are currently no approved therapies that have been shown to modify the course of liver fibrosis. Numerous rodent models have been developed in which liver injury is induced by trauma, toxins, or chronic infections. ${ }^{33-36}$ None of these, however, recapitulate the natural history commonly seen in patients with nonalcoholic (NA) liver disease. Recently, the Stelic animal model (STAM) was introduced. ${ }^{37}$ In this model, designed to replicate the clinically observed, insulin-resistance-driven progression from hepatic steatosis to NA steatohepatitis (NASH), liver fibrosis, and hepatocellular carcinoma, neonatal male mice are treated with a carefully titrated dose of streptozotocin, followed by feeding with a high-fat diet. Fatty liver (FL) is induced by 5 weeks and NASH by 6 weeks in $100 \%$ of the animals. Here, we report the effects of pacritinib in the STAM mouse model, compare these with effects of telmisartan, an 
angiotensin 2-receptor antagonist and PPAR $\gamma$ partial agonist with known antifibrotic effects in rodent models, and present hypotheses on the mechanisms potentially involved.

\section{Methods}

\section{Animal model}

The STAM mouse model has been described previously. ${ }^{37}$ Two-day old male C57BL/6J mice (Japan SLC, Tokyo, Japan) were given a single $200 \mu \mathrm{g}$ subcutaneous dose of streptozotocin. At 4 weeks of age, they were fed a sterilized high-fat diet (HFD32; CLEA, Tokyo, Japan). The sterilized high-fat diet and water were provided ad libitum. Animals were housed and cared for in accordance with the Japanese Pharmacology Society Guidelines for Animal Use ${ }^{38}$ and the study was approved by the SMC Laboratories institutional animal care and use committee. They were maintained in a specific-pathogen-free facility under controlled temperature, humidity, lighting, and air exchange. Pacritinib (CTI BioPharma, Seattle, WA, USA) was suspended in vehicle consisting of $0.5 \% \mathrm{weight} /$ volume methylcellulose $(4,000 \mathrm{cP})$ and $0.1 \%$ volume/volume Tween 80 . Telmisartan (Boehringer Ingelheim, Ingelheim am Rhein, Germany), the positive control, was dissolved in pure water. Groups consisted of eight mice each. The treatment schedule for the vehicle (negative control), pacritinib, and telmisartan is shown in Table 1. Body weight was recorded prior to treatment and then daily. Mice were observed for signs of toxicity, moribundity, and mortality for approximately 60 minutes after each administration. Animals were killed by exsanguination through direct cardiac puncture under ether anesthesia at 9 weeks of age.

\section{Histopathological analysis}

For hematoxylin and eosin (H\&E) staining, sections were cut from paraffin blocks of liver tissue prefixed in Bouin solution and stained with Mayer's hematoxylin (Lillie's modification; Muto Pure Chemicals, Tokyo, Japan) and eosin (Wako Pure Chemicals Industries, Osaka, Japan) solution. NA fatty-liver disease (NAFLD) activity score was calculated according to the criteria of Kleiner et al. ${ }^{39}$

\section{Plasma cytokeratin 18 fragment levels}

Plasma CK18 (M30) level was quantified using the Mouse Cytokeratin 18-M30 enzyme-linked immunosorbent-assay kit (Cusabio, College Park, MD, USA) as per label instructions. Briefly, plasma (1:500 in sample diluent) was added to the wells and incubated at $37^{\circ} \mathrm{C}$ for 2 hours. After removal of liquid, biotin antibody was added and wells incubated at $37^{\circ} \mathrm{C}$ for 1 hour, then washed with buffer three times and incubated with $100 \mu \mathrm{L}$ of HRP-avidin for 1 hour. Enzymatic activity was detected using tetramethylbenzidine substrate and absorbance measured at $450 \mathrm{~nm}$ after a 30 -minute incubation.

\section{Statistical tests}

Statistical analyses were performed using Bonferroni multiple-comparison tests on GraphPad Prism 6 (GraphPad Software, La Jolla, CA, USA). Results are expressed as the means $\pm \mathrm{SD}$.

\section{Results Deaths}

Deaths prior to day 21 in the treatment groups were none in the vehicle group, one in the telmisartan group (day 10) and three in the pacritinib group (days 5, 7, and 10). For animals in the pacritinib group, severe weight loss was noted prior to death, but pathology results did not reveal any unusual findings (eg, vascular occlusion or cerebral bleeding). Based on these results, the $200 \mathrm{mg} / \mathrm{kg}$ pacritinib dose was deemed intolerable and mice treated with $150 \mathrm{mg} / \mathrm{kg}$ thereafter (Table 1). No further deaths occurred.

\section{Weight changes}

There was no significant difference in mean body weight between the vehicle and pacritinib groups on any day. The mean body weight of the positive control (telmisartan) group was significantly lower than that of the vehicle group on day 21 , as assessed by the two-way analysis of variance (ANOVA) Bonferroni test (Figure 1). On the necropsy day (day 0 of week 9), there were no significant differences in mean body weight among any of the treatment groups, as assessed by the one-way ANOVA Bonferroni test (Figure 2A).

Table I Treatment schedule for all groups during weeks 6-9

\begin{tabular}{lllll}
\hline Test substance & Dose $\mathbf{( m g / k g )}$ & Volume $\mathbf{( m g / k g )}$ & Regimens & Death (week) \\
\hline Vehicle & NA & 10 & Oral, twice daily, 6-9 weeks & 9 \\
Pacritinib & 200 & 10 & Oral, twice daily (day 0 to morning of day 10) & 9 \\
& 150 & 10 & Oral, twice daily (afternoon of day 10 to day 21) & 9 \\
Telmisartan & 10 & 10 & Oral, once daily, 6-9 weeks & 9 \\
\hline
\end{tabular}

Abbreviation: NA, not applicable. 


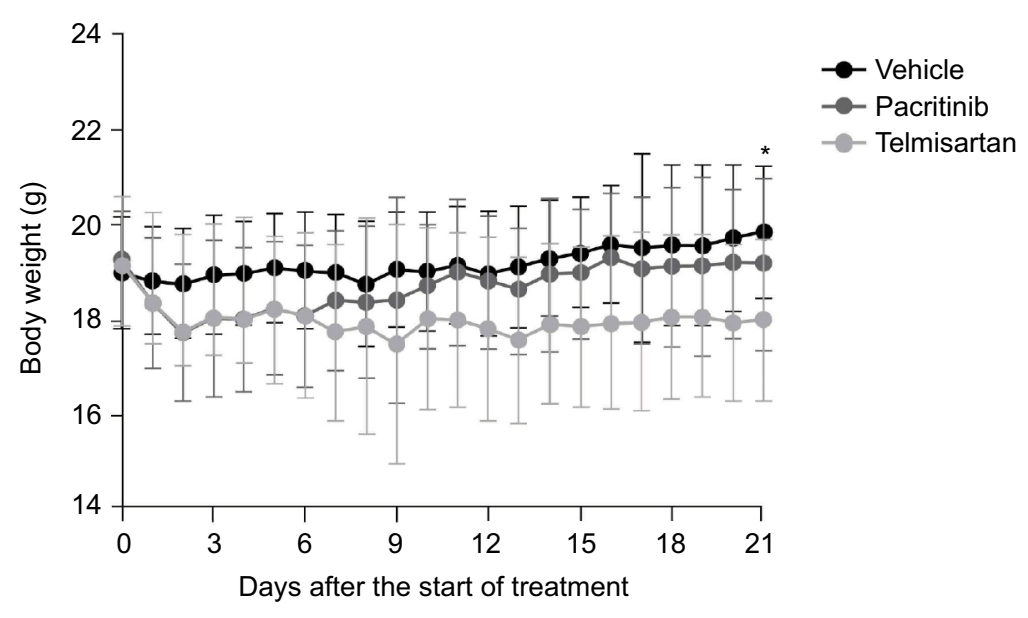

Figure I Body-weight changes in all treatment groups ( $n=8$ per group). ${ }^{*} P<0.05$, telmisartan vs vehicle.

A

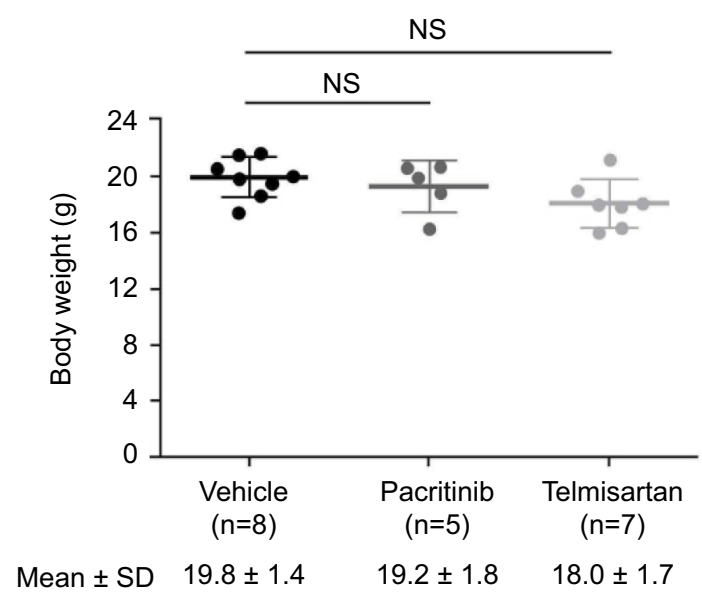

B

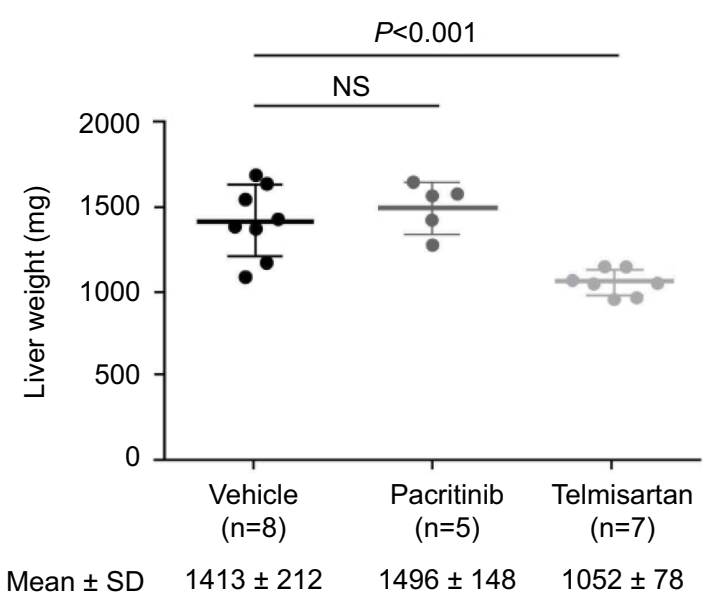

\section{C}

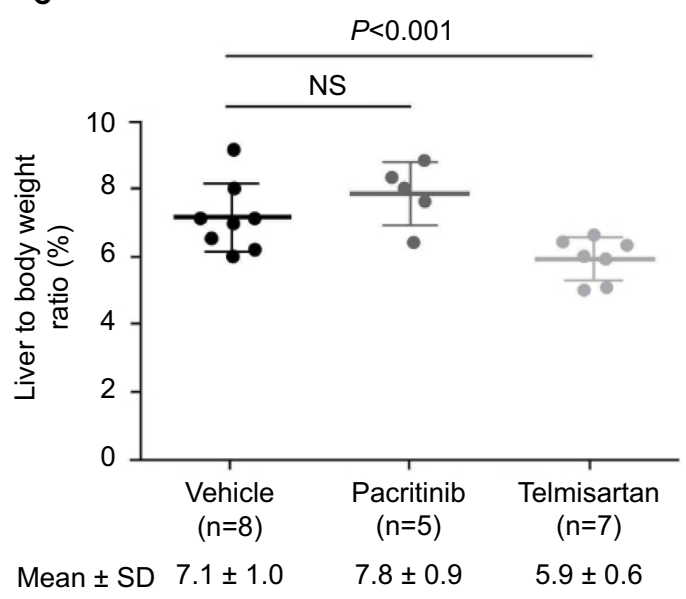

Figure 2 (A) Body weight, (B) liver weight, and (C) liver:body weight ratio on day of death.

Abbreviation: NS, not significant. 
The telmisartan group had significantly decreased mean liver weight $(P<0.001)$ and mean liver:body weight ratio $(P<0.05)$ compared with the vehicle group (Figure 2, B and C), and there were no significant differences between the pacritinib and vehicle groups.

\section{Histological analysis}

Representative micrography of the H\&E-stained liver sections is shown in Figure 3. Liver sections from the vehicle group exhibited severe micro- and macrovesicular fat deposition, hepatocellular ballooning, and inflammatory cell infiltration. The telmisartan group showed a significant $(P<0.01)$ reduction in NAFLD-activity score compared with the vehicle group (Table 2, Figure 4). Although a trend toward lower steatosis and hepatocyte-ballooning scores were noted in the pacritinib group relative to the vehicle group, the difference in NAFLD score between the two groups was not significant. Representative micrography of Sirius red-stained
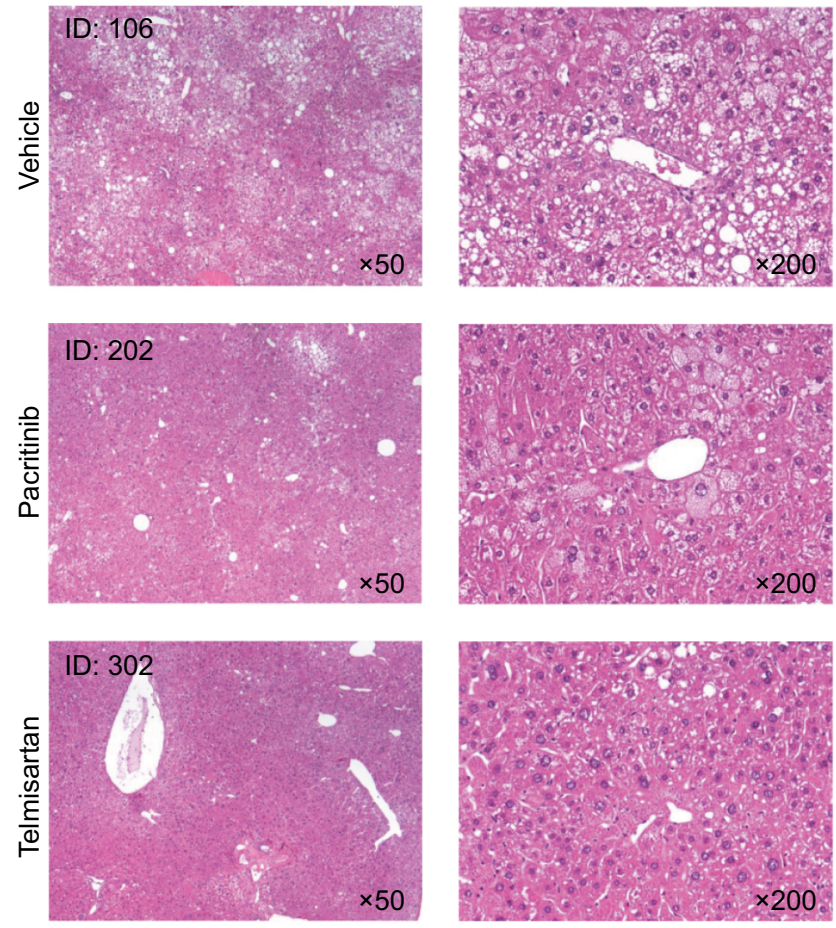

Figure 3 Representative micrography of H\&E-stained liver sections on day of death. Abbreviation: H\&E, hematoxylin and eosin. sections of liver is shown in Figure 5. Liver sections from the vehicle group exhibited collagen deposition in the pericentral region of liver lobules. Both the pacritinib and the telmisartan groups showed significant $(P<0.01$ for pacritinib, $P<0.001$ for telmisartan) decreases in the mean percentage of fibrosis area compared with the vehicle control group $(0.74 \% \pm 0.17 \%$, $0.73 \% \pm 0.12 \%$, and $1.08 \% \pm 0.16 \%$, respectively).

\section{Plasma CKI 8 levels}

On the day of death, mean plasma CK18 M30 levels were 282.9 $\pm 24.2,237.6 \pm 29.5$, and $357.7 \pm 39.7 \mathrm{mIU} / \mathrm{mL}$ for vehicle, pacritinib, and telmisartan, respectively (Figure 6). Relative to vehicle (negative control) CK18 M30 levels were significantly lower $(P<0.05)$ in the pacritinib group and significantly higher $(P<0.001)$ in the telmisartan group.

\section{Discussion}

Pharmacological inhibition of JAK2 has previously been reported to attenuate liver fibrosis in rodent models, although the mechanism has not been fully elucidated. ${ }^{40}$ The observation that pacritinib reduces levels of sIL6, sIL17A, and sIL17F in a system of peripheral blood mononuclear cells, fibroblasts, and endothelial cells that models T-cell-dependent B-cell activation ${ }^{24}$ suggests a number of potential intervention points in addition to JAK2 signaling through which it could exert an anti-inflammatory and/or antifibrotic effect (Figure 7).

IRAK 1 is a serine/threonine kinase that plays a key role in the IL1-IL6 axis ${ }^{41}$ and has a major role in steatosis associated liver cirrhosis and progression to hepatocellular carcinoma. ${ }^{42}$ Knockdown of IRAKI in human hepatic stellate cells (HSCs) results in reductions in the release of inflammatory cytokines associated with local inflammation and promotion of fibrosis. ${ }^{43}$ IRAK1 is critical to signaling by Toll-like receptors activated by fatty acids and other lipid derivatives, and appears to be central to lipid-mediated inflammation. ${ }^{44,45}$ In mouse models of acute and chronic inflammation, IRAK1 deletion dampens inflammatory responses by disfavoring naïve T-cell differentiation into $\mathrm{T}_{\mathrm{H}} 17$ cells, thereby decreasing levels of IL17, the proinflammatory cytokine that plays a pivotal role

Table 2 Nonalcoholic fatty-liver disease-activity score ${ }^{39}$

\begin{tabular}{|c|c|c|c|c|c|c|c|c|c|c|c|c|c|}
\hline \multirow[t]{2}{*}{ Group } & \multirow[t]{2}{*}{$\mathbf{n}$} & \multicolumn{4}{|c|}{ Steatosis } & \multicolumn{4}{|c|}{ Lobular inflammation } & \multicolumn{3}{|c|}{ Hepatocyte ballooning } & \\
\hline & & 0 & I & 2 & 3 & 0 & I & 2 & 3 & 0 & I & 2 & \\
\hline Vehicle & 8 & - & 6 & 2 & - & - & I & 6 & 1 & - & I & 7 & $5.1 \pm 0.6$ \\
\hline Pacritinib & 5 & 3 & 2 & - & - & - & 2 & I & 2 & I & 2 & 2 & $3.6 \pm 1.5$ \\
\hline Telmisartan & 7 & 3 & 4 & - & - & I & 5 & I & - & 2 & I & 4 & $2.9 \pm 1.6$ \\
\hline
\end{tabular}




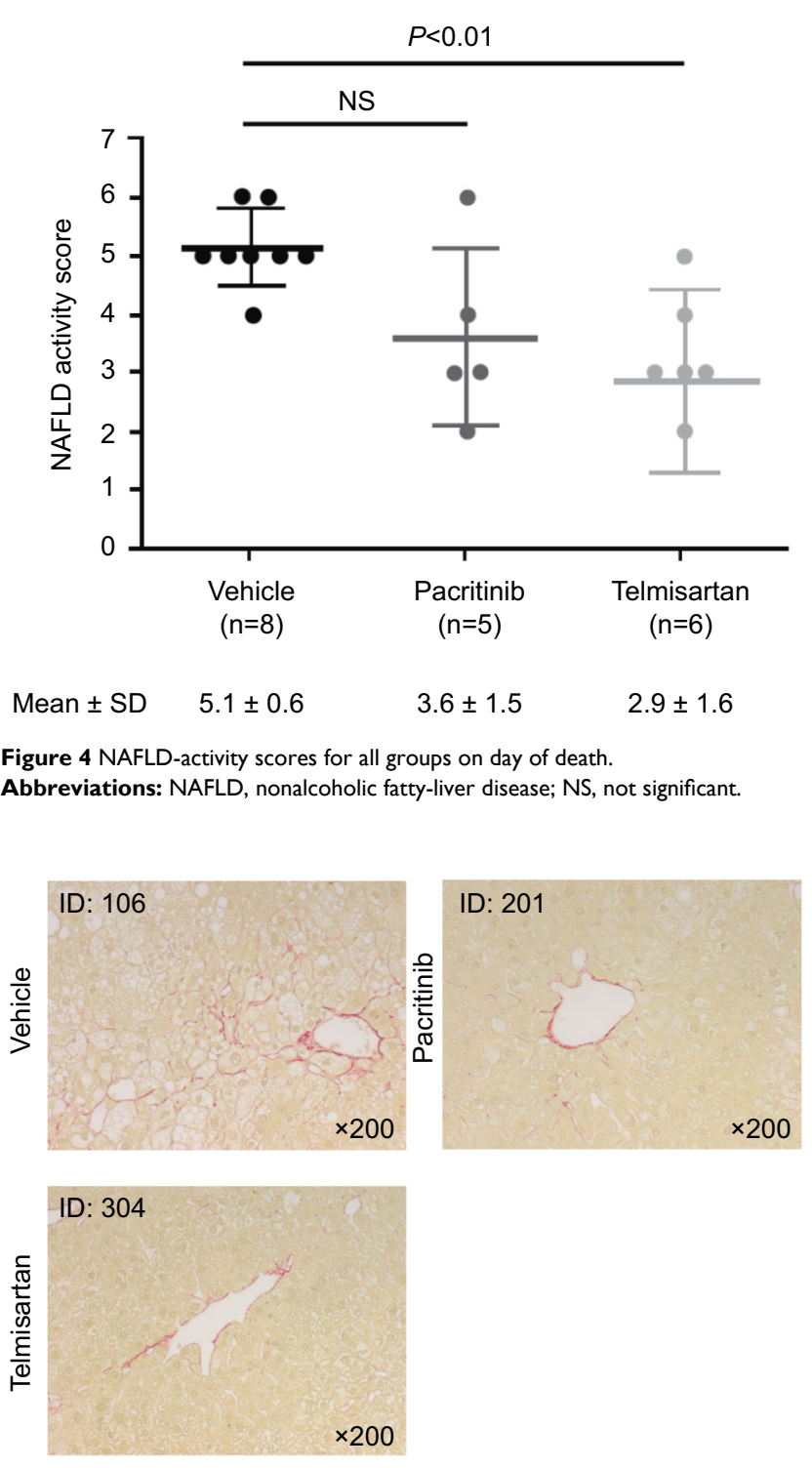

Figure 5 Representative photomicrographs of Sirius red-stained liver sections on day of death.

in HSC activation, which gives rise to $\sim 90 \%$ of myofibroblasts in liver-fibrosis models. ${ }^{46-48}$ Therefore, inhibition of IRAK1 by pacritinib may underlie the observed reduction in IL6 levels, causing a consequent depletion of $\mathrm{T}_{\mathrm{H}} 17$ cells, IL17A, and IL17F.

Pacritinib may also inhibit $\mathrm{T}_{\mathrm{H}}$ 17-cell differentiation through effects on the transcription factor RORC (ROR $\gamma t$ in mice), induction of which depends on full activation of STAT3 in processes dependent upon IRAK1 and JAK2, both of which pacritinib inhibits. Following differentiation, JAK2 associated with the IL23/IL12R $\beta 1$ receptor plays a role in increased IL17A transcription, thus representing another possible intervention point for pacritinib. Finally, pacritinib

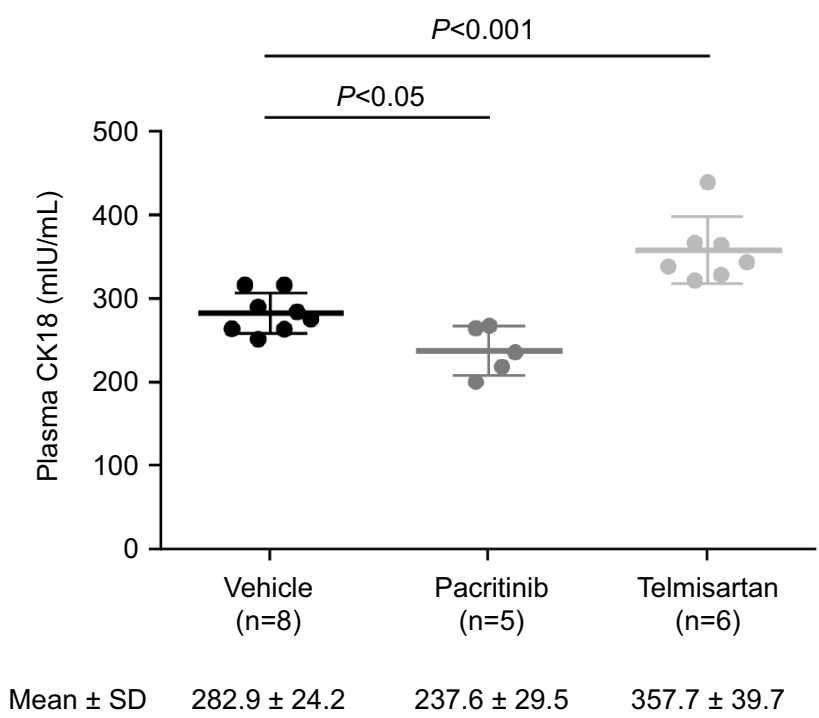

Figure 6 Plasma levels of CKI 8 fragment M30 on day of death.

also inhibits CSF1R kinase, thereby disfavoring the differentiation of monocytes to macrophages, ${ }^{49}$ which promote myofibroblast survival and contribute to the development of liver fibrosis. ${ }^{50,51}$ Notably, controlling macrophage differentiation as an antifibrotic strategy in MF via a different pathway (using recombinant human pentraxin 2) is the subject of ongoing clinical investigation. ${ }^{52}$

The present study investigated whether pacritinib, acting through one or more of these mechanisms, could exert antifibrotic effects in a mouse model that recapitulated the clinical progression commonly seen in human liver disease. In the STAM mouse model, pacritinib had no significant effect on body weight, liver weight, liver:body weight ratio, or NAFLD score relative to vehicle. As such, it did not significantly affect fat accumulation, the inflammatory trigger for liver fibrosis. Nonetheless, it significantly reduced fibrotic area, suggesting inhibition of the inflammatory and subsequent fibrotic response to steatosis. In the same assay, telmisartan, an angiotensin 2 receptor antagonist and PPAR $\gamma$ partial agonist that has demonstrated antifibrotic ${ }^{53}$ and hepatoprotective ${ }^{54}$ activity in rodent models, most likely through downregulation of TGF $\beta$ and suppression of HSC activation, ${ }^{55,56}$ was used as a positive control. In contrast to pacritinib, telmisartan had significant effects on liver weight, liver:body weight ratio, and NAFLD score, in addition to fibrosis area. These results are consistent with a clinical study that reported significantly improved NAFLD and fibrosis scores for telmisartan plus lifestyle modifications relative to lifestyle modifications alone in human patients with NASH. ${ }^{57}$ Differential effects of pacritinib and telmisartan in the STAM 


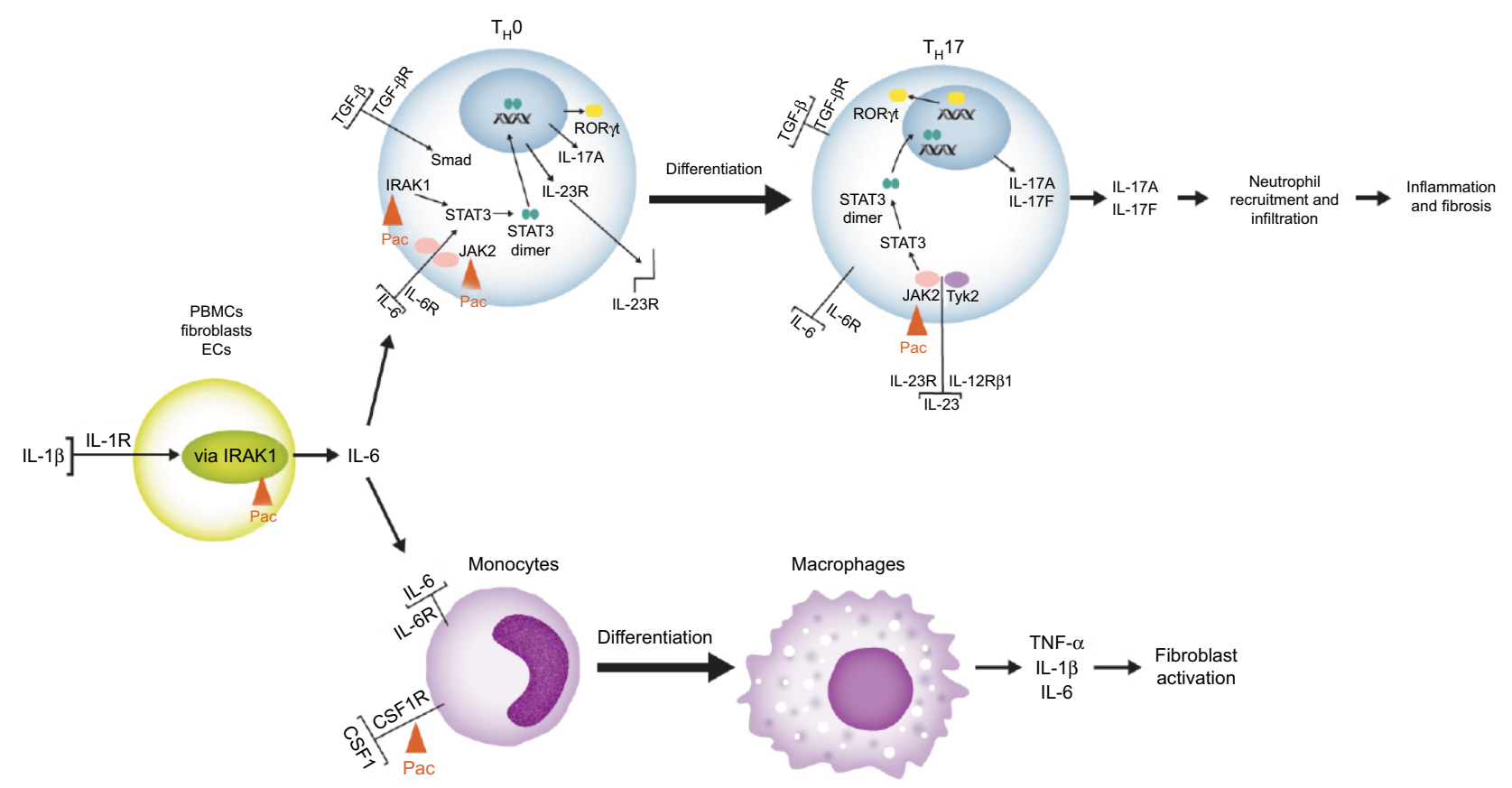

Figure 7 Inflammation pathways and potential pacritinib intervention points in fibrosis.

Notes: In peripheral blood monocytes, fibroblasts and endothelial cells, binding of ILI $\beta$ induces IL6 secretion in a process involving IRAKI. Observed phenotypic reductions in IL6 levels induced by pacritinib may reflect IRAKI inhibition, with downstream effects on inflammation, fibroblast activation, and fibrosis. Upper schema: in naïe T cells, binding of IL6 (together with TGF $\beta$ ) ultimately induces differentiation into $T_{H} 17$ cells. Both JAK2 and IRAKI (both of which pacritinib inhibits) are necessary for STAT3 activation; activated STAT3 dimerizes and translocates to the nucleus, where it binds to DNA, leading to expression of the transcription factor ROR $\gamma$ t, which is critical for $T_{H}$ 17-cell differentiation, and export of IL23R. In $T_{H} 17$ cells, IL23 binds to the IL23R/ILI 2R $\beta$ I receptor to activate STAT3 in another JAK2-dependent process; binding of the STAT3 dimer promotes expression of ILI7A, while binding of ROR $\gamma \mathrm{t}$ promotes the expression of ILI7A and ILI7F, both of which are secreted by $T_{H} 17$ cells, leading to neutrophil recruitment and infiltration and downstream inflammation and fibrosis. Lower schema: IL6 acts as a switch to induce differentiation of monocytes to macrophages rather than dendritic cells; a second critical factor in monocyte differentiation is CSFIR, the kinase activity of which pacritinib also inhibits; macrophages secrete the proinflammatory cytokines TNF $\alpha$, ILI $\beta$, and IL6, which are involved in fibroblast activation.

Abbreviations: Pac, pacritinib; PBMCs, peripheral blood mononuclear cells; ECs, endothelial cells.

model likely reflect the additional mechanism of action, PPAR $\gamma$ partial agonism, associated with telmisartan. This has effects on hepatic fatty oxidation, hepatic lipogenesis, and peripheral as well as hepatic insulin sensitivity. ${ }^{58}$ Finally, the present study examined levels of circulating CK18 fragment in all three groups of animals. Plasma CK18 fragment levels represent a biomarker of the extent of hepatocyte apoptosis, with increased levels predicting clinically observed liver fibrosis ${ }^{59}$ NASH occurrence, and NASH severity. ${ }^{60}$ CK18 levels were significantly reduced relative to vehicle control in animals treated with pacritinib, a finding in line with the significantly reduced extent of liver fibrosis observed by histopathology in this group.

The present pilot translational study has several limitations. A relatively small number of animals was tested, and biomarkers that could link the observed activity of pacritinib to the proposed mechanisms of action were not examined. Further studies are needed to elucidate the pharmacological basis for the effects of pacritinib in liver fibrosis. Bearing these caveats in mind, this is the first study to demonstrate hepatic antifibrotic effects for pacritinib in a nonclinical model of liver disease.

The results of this study lend support to longitudinal assessment of the effect of pacritinib on marrow fibrosis in patients with MF enrolled in upcoming clinical trials, and moreover provide preliminary support to pilot clinical development in liver cirrhosis, along with other fibrotic conditions, such as pulmonary fibrosis and scleroderma.

\section{Acknowledgments}

Medical writing and editorial assistance was funded by CTI BioPharma and provided under the direction of the authors by Nexus Global Group Science.

\section{Disclosure}

SAF and JWS are employed by and have equity ownership in CTI BioPharma. TH and YS are employed by SMC Laboratories. JM has received research funding paid to his institution from Incyte, Roche, Promedior, CTI BioPharma, KaloBios, and Novartis, and has consulted for Incyte and Novartis. 


\section{References}

1. Wynn TA. Common and unique mechanisms regulate fibrosis in various fibroproliferative diseases. J Clin Invest. 2007;117(3):524-529.

2. Akhmetshina A, Palumbo K, Dees C, et al. Activation of canonical Wnt signalling is required for TGF- $\beta$-mediated fibrosis. Nat Commun. 2012;3:735

3. Gabrielli A, Avvedimento EV, Krieg T. Scleroderma. N Engl J Med. 2009;360(19):1989-2003.

4. Zahr AA, Salama ME, Carreau N, et al. Bone marrow fibrosis in myelofibrosis: pathogenesis, prognosis and targeted strategies. Haematologica. 2016;101(6):660-671.

5. Thiele J, Rompcik V, Wagner S, Fischer R. Vascular architecture and collagen type IV in primary myelofibrosis and polycythaemia vera: an immunomorphometric study on trephine biopsies of the bone marrow. Br J Haematol. 1992;80(2):227-234.

6. Agarwal A, Morrone K, Bartenstein M, Zhao ZJ, Verma A, Goel S. Bone marrow fibrosis in primary myelofibrosis: pathogenic mechanisms and the role of TGF- $\beta$. Stem Cell Investig. 2016;3:5.

7. Baxter EJ, Scott LM, Campbell PJ, et al. Acquired mutation of the tyrosine kinase JAK2 in human myeloproliferative disorders. Lancet. 2005;365(9464):1054-1061.

8. Kralovics R, Passamonti F, Buser AS, et al. A gain-of-function mutation of JAK2 in myeloproliferative disorders. $N$ Engl J Med. 2005;352(17):1779-1790.

9. Levine RL, Wadleigh M, Cools $\mathrm{J}$, et al. Activating mutation in the tyrosine kinase JAK2 in polycythemia vera, essential thrombocythemia, and myeloid metaplasia with myelofibrosis. Cancer Cell. 2005;7(4):387-397.

10. Jakafi [package insert]. Wilmington (DE): Incyte Corporation; 2016.

11. Verstovsek S, Kantarjian H, Mesa RA, et al. Safety and efficacy of INCB018424, a JAK1 and JAK2 inhibitor, in myelofibrosis. $N$ Engl $J$ Med. 2010;363(12):1117-1127.

12. Harrison C, Kiladjian JJ, Al-Ali HK, et al. JAK inhibition with ruxolitinib versus best available therapy for myelofibrosis. $N$ Engl J Med. 2012;366(9):787-798

13. Verstovsek S, Mesa RA, Gotlib J, et al. A double-blind, placebo-controlled trial of ruxolitinib for myelofibrosis. NEngl J Med. 2012;366(9): 799-807.

14. Harrison CN, Vannucchi AM, Kiladjian JJ, et al. Long-term findings from COMFORT-II, a phase 3 study of ruxolitinib vs best available therapy for myelofibrosis. Leukemia. 2016;30(8):1701-1707.

15. Verstovsek S, Mesa RA, Gotlib J, et al. Long-term treatment with ruxolitinib for patients with myelofibrosis: 5-year update from the randomized, double-blind, placebo-controlled, phase 3 COMFORT-I trial. J Hematol Oncol. 2017;10(1):55.

16. Hart S, Goh KC, Novotny-Diermayr V, et al. SB1518, a novel macrocyclic pyrimidine-based JAK2 inhibitor for the treatment of myeloid and lymphoid malignancies. Leukemia. 2011;25(11):1751-1759.

17. Hatzimichael E, Tsolas E, Briasoulis E. Profile of pacritinib and its potential in the treatment of hematologic disorders. J Blood Med. 2014;5:143-152.

18. Singer JW, Al-Fayoumi S, Ma H, Komrokji RS, Mesa R, Verstovsek $\mathrm{S}$. Comprehensive kinase profile of pacritinib, a nonmyelosuppressive Janus kinase 2 inhibitor. J Exp Pharmacol. 2016;8:11-19.

19. Komrokji RS, Seymour JF, Roberts AW, et al. Results of a phase 2 study of pacritinib (SB1518), a JAK2/JAK2(V617F) inhibitor, in patients with myelofibrosis. Blood. 2015;125(17):2649-2655.

20. Mascarenhas J, Hoffman R, Talpaz M, et al. Results of the PERSIST-2 phase 3 study of pacritinib (Pac) versus best available therapy (BAT), including ruxolitinib (Rux), in patients (pts) with myelofibrosis (MF) and platelet counts $<100,000 / \mu \mathrm{L}$. Blood. 2016;128(22):LBA5.

21. Mesa RA, Egyed M, Szoke A, Suvorov A, Perkins A, Mayer J. Pacritinib (PAC) vs best available therapy (BAT) in myelofibrosis (MF): 60-week follow-up of the phase III PERSIST-1 trial. J Clin Oncol. 2016;34(15 Suppl):7065.
22. Verstovsek S, Odenike O, Singer JW, Granston T, Al-Fayoumi S, Deeg HJ. Phase 1/2 study of pacritinib, a next generation JAK2/FLT3 inhibitor, in myelofibrosis or other myeloid malignancies. J Hematol Oncol. 2016;9:137.

23. Mesa RA, Vannucchi AM, Mead A, et al. Pacritinib versus best available therapy for the treatment of myelofibrosis irrespective of baseline cytopenias (PERSIST-1): an international, randomised, phase 3 trial. Lancet Haematol. 2017;4(5):e225-e236.

24. Al-Fayoumi S, Watson R, O’Mahony A, Singer JW. Comparative biomarker profiles of pacritinib, momelotinib, pexidartinib, and ruxolitinib using BioMAP Diversity Plus Panel. Eur J Cancer. 2016;69 Suppl $1: \mathrm{S} 136$

25. Hunter CA, Jones SA. IL-6 as a keystone cytokine in health and disease. Nat Immunol. 2015;16(5):448-457.

26. Wynn TA, Ramalingam TR. Mechanisms of fibrosis: therapeutic translation for fibrotic disease. Nat Med. 2012;18(7):1028-1040.

27. Roussel L, Houle F, Chan C, et al. IL-17 promotes p38 MAPK-dependent endothelial activation enhancing neutrophil recruitment to sites of inflammation. J Immunol. 2010;184(8):4531-4537.

28. Jovanovic DV, Di Battista JA, Martel-Pelletier J, et al. IL-17 stimulates the production and expression of proinflammatory cytokines, IL- $\beta$ and TNF- $\alpha$, by human macrophages. J Immunol. 1998;160(7):3513-3521.

29. Bataller R, Brenner DA. Liver fibrosis. JClin Invest. 2005;115(2):209-218.

30. Ekstedt M, Franzen LE, Mathiesen UL, et al. Long-term follow-up of patients with NAFLD and elevated liver enzymes. Hepatology. 2006;44(4):865-873.

31. Younossi ZM, Koenig AB, Abdelatif D, Fazel Y, Henry L, Wymer M. Global epidemiology of nonalcoholic fatty liver disease: meta-analytic assessment of prevalence, incidence, and outcomes. Hepatology. 2016;64(1):73-84.

32. Ellis EL, Mann DA. Clinical evidence for the regression of liver fibrosis. J Hepatol. 2012;56(5):1171-1180.

33. Yanguas SC, Cogliati B, Willebrords J, et al. Experimental models of liver fibrosis. Arch Toxicol. 2016;90(5):1025-1048.

34. Iredale JP. Models of liver fibrosis: exploring the dynamic nature of inflammation and repair in a solid organ. J Clin Invest. 2007;117(3):539-548.

35. Liedtke C, Luedde T, Sauerbruch T, et al. Experimental liver fibrosis research: update on animal models, legal issues and translational aspects. Fibrogenesis Tissue Repair. 2013;6(1):19.

36. Wynn TA. Fibrotic disease and the $\mathrm{T}(\mathrm{H}) 1 / \mathrm{T}(\mathrm{H}) 2$ paradigm. Nat Rev Immunol. 2004;4(8):583-594.

37. Fujii M, Shibazaki Y, Wakamatsu K, et al. A murine model for nonalcoholic steatohepatitis showing evidence of association between diabetes and hepatocellular carcinoma. Med Mol Morphol. 2013;46(3): 141-152.

38. Ohno Y. [Animal experiments recommended by the Japanese Pharmacological Society (evaluation and decrease of pain): introduction and new guidelines for animal experiments for the Japanese Pharmacological Society]. Nihon Yakurigaku Zasshi. 2007;129(1):5-9. Japanese.

39. Kleiner DE, Brunt EM, van Natta M, et al. Design and validation of a histological scoring system for nonalcoholic fatty liver disease. Hepatology. 2005;41(6):1313-1321.

40. Granzow M, Schierwagen R, Klein S, et al. Angiotensin-II type 1 receptor-mediated Janus kinase 2 activation induces liver fibrosis. Hepatology. 2014;60(1):334-348.

41. Weber A, Wasiliew P, Kracht M. Interleukin-1 (IL-1) pathway. Sci Signal. 2010;3(105):cm1

42. Ye ZH, Gao L, Wen DY, He Y, Pang YY, Chen G. Diagnostic and prognostic roles of IRAK1 in hepatocellular carcinoma tissues: an analysis of immunohistochemistry and RNA-sequencing data from the cancer genome atlas. Onco Targets Ther. 2017;10:1711-1723.

43. Chen Y, Zeng Z, Shen X, Wu Z, Dong Y, Cheng JC. MicroRNA-146a-5p negatively regulates pro-Inflammatory cytokine secretion and cell activation in lipopolysaccharide stimulated human hepatic stellate cells through inhibition of Toll-like receptor 4 signaling pathways. Int J Mol Sci. 2016;17(7):E1076 
44. Ahmad R, Shihab PK, Thomas R, et al. Increased expression of the interleukin-1 receptor-associated kinase (IRAK)-1 is associated with adipose tissue inflammatory state in obesity. Diabetol Metab Syndr. 2015;7:71.

45. Lee JY, Hwang DH. The modulation of inflammatory gene expression by lipids: mediation through Toll-like receptors. Mol Cells. 2006;21(2):174-185.

46. Maitra U, Davis S, Reilly CM, Li L. Differential regulation of Foxp3 and IL-17 expression in CD4 T helper cells by IRAK-1. J Immunol. 2009;182(9):5763-5769.

47. Mederacke I, Hsu CC, Troeger JS, et al. Fate tracing reveals hepatic stellate cells as dominant contributors to liver fibrosis independent of its aetiology. Nat Commun. 2013;4:2823.

48. Tan Z, Qian X, Jiang R, et al. IL-17A plays a critical role in the pathogenesis of liver fibrosis through hepatic stellate cell activation. J Immunol. 2013;191(4):1835-1844.

49. Pixley FJ, Stanley ER. CSF-1 regulation of the wandering macrophage: complexity in action. Trends Cell Biol. 2004;14(11):628-638.

50. Pradere JP, Kluwe J, de Minicis S, et al. Hepatic macrophages but not dendritic cells contribute to liver fibrosis by promoting the survival of activated hepatic stellate cells in mice. Hepatology. 2013;58(4): 1461-1473.

51. Wynn TA, Vannella KM. Macrophages in tissue repair, regeneration, and fibrosis. Immunity. 2016;44(3):450-462.

52. Verstovsek S, Mesa RA, Foltz LM, et al. PRM-151 in myelofibrosis: durable efficacy and safety at 72 weeks. Blood. 2015;126(23):56.
53. Nakagami H, Kiomy Osako M, Nakagami F, et al. Prevention and regression of non-alcoholic steatohepatitis (NASH) in a rat model by metabosartan, telmisartan. Int J Mol Med. 2010;26(4):477-481.

54. Tamaki Y, Nakade Y, Yamauchi T, et al. Angiotensin II type 1 receptor antagonist prevents hepatic carcinoma in rats with nonalcoholic steatohepatitis. J Gastroenterol. 2013;48(4):491-503.

55. Halici Z, Bilen H, Albayrak F, et al. Does telmisartan prevent hepatic fibrosis in rats with alloxan-induced diabetes? Eur J Pharmacol. 2009;614(1-3):146-152.

56. Jin H, Yamamoto N, Uchida K, Terai S, Sakaida I. Telmisartan prevents hepatic fibrosis and enzyme-altered lesions in liver cirrhosis rat induced by a choline-deficient L-amino acid-defined diet. Biochem Biophys Res Commun. 2007;364(4):801-807.

57. Alam S, Kabir J, Mustafa G, Gupta U, Hasan SK, Alam AK. Effect of telmisartan on histological activity and fibrosis of non-alcoholic steatohepatitis: a 1-year randomized control trial. Saudi J Gastroenterol. 2016;22(1):69-76.

58. Benson SC, Pershadsingh HA, Ho CI, et al. Identification of telmisartan as a unique angiotensin II receptor antagonist with selective PPAR $\gamma$ modulating activity. Hypertension. 2004;43(5):993-1002.

59. Mandelia C, Collyer E, Mansoor S, et al. Plasma cytokeratin-18 level as a novel biomarker for liver fibrosis in children with nonalcoholic fatty liver disease. J Pediatr Gastroenterol Nutr. 2016;63(2):181-187.

60. Feldstein AE, Wieckowska A, Lopez AR, Liu YC, Zein NN, McCullough AJ. Cytokeratin-18 fragment levels as noninvasive biomarkers for nonalcoholic steatohepatitis: a multicenter validation study. Hepatology. 2009;50(4):1072-1078.
Journal of Experimental Pharmacology

\section{Publish your work in this journal}

The Journal of Experimental Pharmacology is an international, peerreviewed, open access journal publishing original research, reports, reviews and commentaries on all areas of laboratory and experimental pharmacology. The manuscript management system is completely online and includes a very quick and fair peer-review system.

\section{Dovepress}

Visit http://www.dovepress.com/testimonials.php to read real quotes from published authors. 